\title{
Multigraph decomposition into multigraphs with two underlying edges
}

\author{
Miri Priesler $^{1}$ and Michael Tarsi ${ }^{2}$ \\ ${ }^{1}$ Engineering Department, Ruppin Academic Centre, Emek Hefer 40250, Israel \\ ${ }^{2}$ School of Computer Science, Tel-Aviv University, Tel-Aviv 69978, Israel
}

Due to some intractability considerations, reasonable formulation of necessary and sufficient conditions for decomposability of a general multigraph $G$ into a fixed connected multigraph $H$, is probably not feasible if the underlying simple graph of $H$ has three or more edges. We study the case where $H$ consists of two underlying edges. We present necessary and sufficient conditions for $H$-decomposability of $G$, which hold when certain size parameters of $G$ lies within some bounds which depends on the multiplicities of the two edges of $H$. We also show this result to be "tight" in the sense that even a slight deviation of these size parameters from the given bounds results intractability of the corresponding decision problem.

Keywords: Decomposition, Multigraph, NPC

\section{Extended Abstract}

Given two graphs $H$ and $G$, an $H$-decomposition of $G$ is a partition of the edge set of $G$ into disjoint isomorphic copies of $H$. The study of Graph decomposition started back at the mid $19^{t h}$ century, with the seminal concept of Steiner triple systems Steiner (1853), and has since become the subject of some hundreds of research papers, with active research still carried out today. R. Wilson's fundamental theorem Wilson (1976) states that for any fixed graph $H$ there exists an $H$-decomposition of the complete graph $K_{n}$ if the obvious necessary divisibility conditions hold and $n$ is large enough. A considerable amount of research was indeed devoted to thoroughly studying the existence of $H$ decompositions of complete graphs for specific graphs $H$, such as: some small graphs, complete graphs, complete multipartite graphs, paths and cycles (a finite problem for every fixed graph $H$, in light of Wilson's theorem). For a review of methods and results see e.g. Bermond and Sotteau (1975) and Bosak (1990).

Hopes for similar accurate results where $H$ decomposition of a general graph $G$ is considered are slim, due to the following negative result:

Theorem 1.1 Deciding whether there exists an $H$-decomposition of an input graph $G$ is NP-complete for any fixed simple graph $H$ which contains a connected component with at least 3 edges

The above was conjectured by I. Holyer Holyer (1981) on 1981 and proved sixteen years later in Dor and Tarsi (1997). On the other hand, the existence of a polynomial time algorithm to decide $\mathrm{H}$ decomposability of an input $G$, where every component of $H$ consists of at most two edges was proved (though not in terms of an explicit necessary and sufficient condition) in Bryś and Lonc (1995). 
In this research we study Multigraph decomposition, that is the case where multiple edges are allowed in both graphs $H$ and $G$. Although Theorem 1.1 was not (yet?) generalized to multigraphs, a graph decomposition decision problem most probably remains at least as hard when extended to multigraphs. Furthermore, we have managed to prove the following intractability results Priesler and Tarsi (2002b):

Theorem 1.2 Deciding the decomposability of an input multigraph $G$ with a constant multiplicity $\lambda$ on all its edges, into the star $K_{1, t}$ is NP-Complete for every fixed $\lambda$ and $t \geq 3$.

Theorem 1.3 Deciding H-decomposability of an input multigraph $G$ into any fixed multistar (a multigraph whose underlying simple graph is $K_{1, t}$, with any sequence of positive multiplicities on its $t$ edges) with at least three underlying edges, is NP-Complete.

In an attempt to find the conditions for decomposability of a general "input" multigraph $G$ into a "fixed" connected multigraph $H$, serious hopes for results are limited, in light of the theorems above, to the case were $H$ consists of two underlying edges.

Quite surprisingly we found out this limited setting to be rather involved, producing somewhat unexpected results: In a previous result Priesler and Tarsi (2004), we considered the simplest case, where $H=S^{1,2}$ is a multigraph on anderlying $K_{1,2}$ with multiplicity 1 on one edge and 2 on the other, and $G$ is a multigraph on any underlying simple graph with a constant multiplicity $\lambda$ on all its edges. We gave necessary and sufficient condition for such a decomposition to exist if $\lambda \neq 2$ and $\lambda \neq 5$. We also showed that similar conditions for $\lambda=2$ and for $\lambda=5$ do most probably not exist, by proving the corresponding decision problems to be $N P$-complete.

In our article we investigate the decomposition of a general multigraph $G$ into $S^{\alpha, \beta}$ - an underlying $K_{1,2}$ with multiplicities $\alpha$ and $\beta$. We show some necessary divisibility conditions to be also sufficient if certain size parameters of $G$ lie between certain bounds which depend on $\alpha, \beta$ and $\frac{\alpha}{\beta}$. The following theorem summarizes this main result:

Theorem 1.4 Let $\alpha$ be a larger integer than an integer $\beta$ and let $\varepsilon$ be any constant $0<\varepsilon<1-\frac{\beta}{\alpha}$, then there exist $\lambda_{0}(\alpha, \beta, \varepsilon)$ and $M_{0}(\alpha, \beta, \varepsilon)$ such that for every $\lambda>\lambda_{0}$

If $G=(V, E)$ is

- Any connected graph, other than an odd regular tree, or

- An odd regular tree where $|E| \geq M_{0}$, and

- $w: E \rightarrow\left[\lambda, \frac{\alpha}{\beta}(1-\varepsilon) \lambda\right]$, represents the multiplicity function of the edges (clearly integer values)

then $(G, w)$ admits an $S^{\alpha, \beta}$-decomposition if and only if

1. $\sum_{e \in E} w(e)$ is divisible by $\alpha+\beta$

2. if $G$ is either a path, or an even circuit, then $\alpha \sum_{e \text { is odd }} w(e) \equiv \beta \sum_{e \text { is even }} w(e)\left(\bmod \alpha^{2}-\beta^{2}\right)$.

Finally we show this result to be "best possible" in the sense that the corresponding decision problem becomes $N P$-complete when the relevant size requirements are not met. 


\section{References}

N. Alon. A note on the decomposition of graphs into isomorphic matchings, hung. Acta Math, 42: 221-223, 1983.

J. C. Bermond and D. Sotteau. Graph decomposition and G-designs. Proc. of the 5th British combinatorial conference. Aberdeen, pages 53-72, 1975.

A. Bialostocki and Y. Roditty. $3 K_{2}$-decomposition of a graph, hungar. Acta Math. Acad. Sci., 40:201-208, 1982.

A. Bondy. Perfect path double covers of graphs. J. of Graph Theory, 14:259-272, 1990.

J. Bosak. Decompositions of Graphs. Kluwer Academic Publishers, 1990.

A. E. Brouwer and R. M. Wilson. The decomposition of graphs into ladder graphs. Technical report, Mathematisch Centrum (zn 97) Amsterdam, 1980.

K. Bryś and Z. Lonc. A complete solution of a Holyer problem. 4th Twente Workshop on graph and combinatorial optimization, University of Twente, Enschede, The Netherlands, June 1995.

Y. Caro. Decomposition and partition of trees into isomorphic subtrees. Master's thesis, Tel-Aviv University, 1985.

E. Cohen and M. Tarsi. NP-completeness of graph decomposition problems. J.Complexity, 7:200-212, 1991.

D. Dor and M. Tarsi. Graph decomposition is NP-complete: proof of Holyer's conjecture. SIAM J. Comput., 26:1166-1187, 1997.

O. Favaron, Z. Lonc, and M. Truszczynski. Decomposition of graphs into graphs with three edges. Ars Combinatoria, 20:125-146, 1985.

M. R. Garey and D. S. Johnson. Computers and Intractability, a guide to the theory of NP-completeness. W. H. Freeman and Company, San Francisco, 1979.

T. Gustavsson. Decompositions of large graphs and digraphs with high minimum degree. PhD thesis, Univ Stockholm, 1991.

I. Holyer. The complexity of graph theory problems. PhD thesis, Churchill College, Cambridge, 1980.

I. Holyer. The NP-completeness of some edge partition problems. Siam J. Comput., 10:713-717, 1981.

D. G. Kirkpatrick and P. Hell. On the completeness of a generalized matching problem. 10th annual ACM Sumposium on Theory of Computing Machinery, New-York, pages 240-245, 1978.

Z. Lonc. Edge decomposition into isomorphic copies of $s K_{1,2}$ is polynomial. J. Combin. Theory Ser. B, 69:164-182, 1997.

S. Masuyama and S. L. Hakimi. Edge packing in graphs. preprint. 
M. Priesler and M. Tarsi. On the decomposition of graphs into copies of $P_{3} \cup t K_{2}$. Ars Combinatoria, 35:325-333, 1993.

M. Priesler and M. Tarsi. Multigraph decomposition into multigraphs with two underlying edges. Preprint, 2002a.

M. Priesler and M. Tarsi. Multigraph decomposition into stars and into multistars. Preprint, 2002b.

M. Priesler and M. Tarsi. On some multigraph decomposition problems and their computational complexity. Discrete Mathematics, 281:247-254, 2004.

J. Steiner. Combinatorische aufgabe. Z. Reine Angew MAth, 45:181-182, 1853.

R. Wilson. Decomposition of a complete graph into subgraphs isomorphic to a given graph. Proc. 5th British Combin. Conf., Aberdeen 1975, Utilitas Math, Winnipeg, pages 647-659, 1976. 